\title{
Aus der geburtshilflich-gynäkologischen Klinik in Basel.
}

\section{Bericht iiber die vom Mai 1887 bis März 1894 in der Basler Franenklinik ausgefiihrten Prolaps- operationen, speciell iiber die Kolporrhaphia ant. duplex.}

Von

Dr. E. Niebergall,

Volontäraret der Klinik.

Wie auf dem Gebiete der inneren Medicin in unserer rasch vorwärts strebenden Zeit ein Heilmittel nach dem anderen angepriesen wird, um oft leider nach kurzer Zeit als nicht den Anforderungen entsprechend zu verschwinden, so zeigt auch in der Chirurgie und operativen Gynäkologie manche als vortrefflich angepriesene Operation Mängel und Nachtheile, die deren Anwendung illusorisch macht. Es hängt eben sehr häufig der Werth einer Operation nicht ab von dem momentan oft glänzenden Erfolg, sondern davon, ob sie dauernde Heilung von dem zu bekämpfenden Leiden schafft. Einen besseren Beweis für die Richtigkeit dieser Behauptung könnte man wohl kaum finden, als die Anführung der verschiedenen Prolapsoperationen, von deren viele, Anfangs glänzende Erfolge aufweisend, bald nach kurzer Zeit verlassen wurden, da die nach längerer oder kürzerer Zeit eintretenden Recidive ihre Nutzlosigkeit erwiesen.

Wichtig ist daher vor allen Dingen eine nach einigen Jahren vorzunehmende Nachprüfung über den Erfolg der Operation, wie solche jetzt aus den verschiedenen Kliniken über die Brauchbarkeit der jeweilen angewandten Operationsmethoden vorliegen. So aus den Kliniken von Berlin, Breslau, Dresden, Kiel, Freiburg, 
362 Niebergall, Bericht übes d. rom Mai 1887 bis März 1894 in d.

Halle u. a., deren Heilungen zwischen 56 und 92 pCt. schwanken. In neuester Zeit veröffentlichte Thieme einen Bericht, über die Erfolge an der Halleschen Frauenklinik; der 61,8 pCt. Heilungen aufweist.

Für die Basler Klinik war eine nochmalige Untersuchung des Erfolges der vorgenommenen Prolapsoperationen von besonderem Interesse, als es galt, die Zweckmässigkeit und Dauerhaftigkeit der von Prof. Fehling eingeführten Kolporrhaphia anterior duplex zu controlliren. Mit Erlaubniss des Herm Prof. Bumm unternahm ich es, das vorhandene Material zu bearbeiten und wenn auch leider nur ca. 70 pCt. der operirten Franen zur Untersuchung kamen, so lässt sich doch aus den Resultaten mancher Schluss über die Vortheile der einen oder anderen Operation ziehen.

Schon im Jahre 1890 hat Rossier in diesem Archiv (Bd. 38, Hft. 3) die Operation beschrieben, doch sagt er dann am Schluss: "Zur Aufstellung einer Statistik des Fehling'schen Verfahrens gegenïber anderen müssen erst noch weitere Beobachtungen gemacht werden".

Da inzwischen seit Einführung der Operation 7 Jahre verffossen sind und ca. 50 Fälle an der Basler Klinik wegen Prolaps durch die Kolporrhaphia anterior duplex, 60 Fälle durch die Kolporrhaphia anterior simplex behandelt wurden, so dürfte die Besprechung derselben wohl gestattet sein.

Handelt es sich um einseitigen Vorfall der vorderen oder hinteren Scheidenwand, so haben wir es in der grossen Mehrzahl der Fälle mit dem Prolapsus vaginae anterioris zu thun, bei erhaltenem Damme ist er überhaupt der einzig mögliche. Trotz dieses Umstandes wurde der Kolporrhaphia anterior meist nur eine untergeordnete Bedeutung bei der Operation des Prolapses zugeschrieben.

Fehling ${ }^{1}$ ) hatte nun die Erfahrung gemacht, dass gerade die junge, median gelegene Narbe durch die sich füllende Blase, durch die Athembewegungen einem ewigen Wechsel zwischen Bewegung und Ruhe unterworfen ist; dadurch kommt, zumal bei breiter Anfrischung, leicht eine theilweise Dehiscenz zu Stande oder es giebt selbst bei guter primärer Heilung später die Narbe nach. Er lässt daher die Mitte in der Breite von $1,0-1,5 \mathrm{~cm}$ stehen und frischt zwei seitliche, ea. $3 \mathrm{~cm}$ breite Ovale an, deren Längsaxe etwas schräg von unten (Urethra) nach oben gegen die Portio läuft. Der

1) Lehrbuch der Frauenkrankheiten von H. Fehling. S. 281 If. 
Umstand, dass der äussere Rand der Anfrischung etwas länger wird als der innere, lässt sich bei der Naht leicht ansgleichen. Anfrischung, Naht ete. sind wie sonst. Auf der Seite werden zuweilen grössere Venenplexus getroffen, die man unterbindet.

Die Fälle, bei denen die Kolporrhaphia anterior duplex zur Anwendung kam, waren meist sehr starke und grosse Vorfälle, da bei den kleineren das Ausschneiden zweier Schleimhautlappen nicht gut möglich ist. Fast immer wurde der Kolporrhaphie die Amputatio portionis vorausgeschickt. Die Kolporrhaphia posterior oder die Kolpoperineorrbaphie nach Hegar erfolgte in der Mehrzahl der Fälle in einer zweiten Sitzung nach ca. 14 Tagen, um die oft bei den einzeitig ausgeführten Operationen entstehende Spannung zu vermeiden und den Geweben besser Zeit zur Rückbildung zu geben. Als Nahtmaterial wurde fast ausschliesslich Silberdraht mit Catgutnähten zur Adaption dazwischen verwandt.

Von den nun in dem Zeitraum von Mai 1887 bis März 1894 operirten 110 Prolapsfällen wurden 50 nach der Fehling'schen Methode operirt. Von diesen stellten sich 16 zur Controlle. 18 gaben schriftlich Auskunft über den Erfolg der Operation.

Es betrafen diese 34 Fälle:

Vorfall des Uterus allein . . . . . . 0 Fall

Vorfall der Vagina allein . . . . . . 8 Fälle

Vorfall von Uterus und Vagina .. . . $25 n$

Vorfall von Uterus, Vagina und Anus . . 1 Fall.

Diese Fälle vertheilen sich wie folgt:

I. Uterus

II. Vagina.

1. Descensus vaginae ant. et post. . . . 4

2. Prolapsus vaginae ant. et post. . . . 4

III. Uterus und Vagina:

1. Prolapsus uteri et vag. totalis . . . . 12

2. Prolapsus uteri et descensus vaginae. . 10

3. Descensus uteri et prolapsus vaginae . 2

4. Descensus uteri et descensus vaginae . 1

IV. Prolapsus nteri, vaginae et ani . . . . . 1

Von den 16 controllirten waren vollständig geheilt geblieben. . . . . . . 14 ungeheilt . . . . . . . . . . . . . 2

Diese letzteren zwei betreffen. beide Fälle von sehr starkem Prolapsus uteri et vaginae totalis. 
364 Niebergall, Bericht über d. vom Mai 1887 bis März 1894 in d.

Von den 18, die schriftlich geantwortet hatten, waren nach ihrer Angabe geheilt geblieben . . . . . . . . . . 15 ungeheilt . : . . . . . . . . . . . 3 und zwar

1 Fall von Descensus uteri et prolapsus vaginae,

$1 "$ "Descensus uteri et vaginae,

$1 n "$ Descensus vaginae anterior. et posterior.

Von den Geheilten hatten 2 normal geboren, während $2 \mathrm{mal}$ die Entbindung die Ursache des Recidives war, in einem Falle allerdings, nachdem nach 2 maligem Abort bei der darauffolgenden Geburt ein schwerer Forceps angelegt worden war.

Die meisten der geheilt gebliebenen gaben an, dass sie sich trotz oft schwerer Arbcit (auf dem Lande und in der Fabrik) ganz wohl fühlten und dass auch die Menses wieder regelmässiger seien.

Fassen wir nun diese Angaben zusammen, so haben wir von 34 Operirten 29 geheilt geblieben $=85,29$.

Wie schon oben angeführt, war $2 \mathrm{Mal}$ die Geburt die Ursache des Recidivs, doch haben auch 2 Frauen normale Entbindungen durehgemacht, eine davon ist sogar wieder im 4. Monat der Gravidität, ohne dass eine neue Senkung eingetreten wäre.

Betrachten wir nun die Fälle, meist leichterer Natur, wo die Kolporrhaphia anterior simplex ausgeführt wurde (neben Portioamputation und Kolpoperineorrhaphie) so ergiebt sich Folgendes:

Zur Controlle kamen . . . . . . 14

Schriftlich gaben Bericht $\frac{. \quad . \quad .22}{\text { Total } .36}$

und zwar waren

I. Vorfall des Uterus allein. . . . . . . . 0

II. Vorfall der Vagina allein . . . . . . . . 23

III. Vorfall von Uterus und Vagina. . . . . . 13

Davon waren

II. Bei Vorfall der Vagina allein:

1. Descensus vaginae ant. et post. . . . 8

2. Prolapsus vaginae anterior..... . 3

3. Prolapsus vaginae anterior et post. . . 12

III. Bei Vorfall von Vagina und Uterus:

1. Prolapsus uteri et vaginae totalis . . . 4

2. Prolapsus uteri et vaginae incompletus . 1

3. Prolapsus uteri et descensus vaginae. . 5

4. Descensus uteri et prolapsus vaginae . 3 
Basler Frauenkl. ausgef. Prolapsoperationen, spec. Kolporth. ant. duplex. 365

Geheilt geblieben waren von den 14 controllirten . 10

Ungeheilt . . . . . . . . . . . . . . . . 4

Zwei von diesen vier Fällen betrafen Prolapsus vaginae ant. et post.; zwei andere Descensus vaginae ant. et post.

Geschrieben hatten 22 Frauen, davon waren mit Erfolg operirt 18; hatten ein Recidiv ebenfalls $4 ; 3$ von diesen letzteren hatten an Descensus vaginae anterior et posterior gelitten, 1 an Prolapsus uteri et vaginae totalis.

Von diesen 36 Frauen hatten geboren 7 , und war in 2 Fällen die Geburt die Urșache des Recidivs, während in 5 Fällen eine dauernde Heilung, 1 Mal sogar trotz zweimaliger Geburt zu verzeichnen ist.

Wir haben also von den 36 operirten Fällen als geheilt anzusehen $28=77,77$ pCt. als ungeheilt $8=22,22$ pCt.

Zusammen betrachtet haben wir bei den 70 controllirten Prolapsoperationen 57 Heilungen, also 81,53; im Ganzen ein schönes Resultat, besonders, wemn man berücksichtigt, dass 7 von diesen Frauen wieder geboren haben, eine sogar 2 Mal, ohne dass ein Recidiv eingetreten wäre. Ausserdem waren es fast alle Franen, die sich zu Hause nicht schonen konnten, sondern ihren Geschäften nachgehen mussten und theilweise sogar schwere Arbeit verrichteten.

Vergleichen wir aber die Resultate bei den durch die Kolporrhaphia anterior duplex und den durch die Kolporrhaphia anterior simplex Operirten, so sind sie bei den ersteren entschieden günstiger.

Erstens haben wir 85,29 pCt. Heilungen gegen 77,77 pCt. Noch mebr in die Augen springend ist aber der Vortheil, wemn man bedenkt, dass gerade durch die. Kolporrhaphia anterior duplex immer nur die schweren Fälle operirt wurden, wo die Grösse des Prolapses es gestattete, 2 Schleimhautlappen zu excidiren, und es betrafen auch die Recidive bei dieser Operationsmethode zwei schwere Fälle von Vorfall von Uterus und Vagina und nur bei dem einen handelte es sich um einen totalen Scheidenvorfall allein; die ungeheilt gebliebenen Prolapse bei der Kolporrhaphia anterior simplex waren 3 einfache Scheidenvorfälle und nur einer ein Vorfall von Vagina und Uterus.

Auch fiel mir bei der Untersuchung auf, dass bei den nach Fehling operirten Frauen die vordere Scheidenwand nur in zwei 
Fällen etwas sichtbar war, während bei den nach der alten Kolporrhaphia anterior simplex operirten oft ein kleiner Theil der vorderen Scheidenwand beim Pressen hervortrat, ohne dass man jedoch von einem Recidive hätte sprechen können.

Vergleichen wir nun noch die Resultate aus den Kliniken von Basel und Halle, um daraus ein Ergebniss für die 2 verschiedenen Operationsmethoden, Kolporrhaphia ant. duplex and simplex, zu gewinnen, so führt Thieme unter den 59 Controllirten 30 an, bei denen in der Sprechstunde nur ein Recidiv der vorderen Wand constatirt wurde und zwar bei den durch

I. Kolporrhaphia ant. Operirten . . . . . . . . 1

II. Kolpoperineorrhapie Operirten . . . . . . . . . . . . 4

III. Kolporrhaphia ant. und Kolpopernieorrhaphie Operirten 16

IV. Kolporrhaphia ant. post., Dammplastik und Amputatio portionis supravaginalis . . . . . . . . . . 6

V. Kolporrhaphia ant. post., Dammplastik und Amputatio portionis supravaginalis . . . . . . . . . . 3

Also im Ganzen ein erheblicher Procentsatz aller Operirten. Nicht angeführt sind hier diejenigen, wo vordere und hintere Wand prolabirt waren.

Bei unseren Fällen waren yon den 4 Recidiven nach Kolporrhaphia anterior simplex auch 2 Frauen, bei denen nur die vordere Wand wieder vorgefallen war, 2 bei denen vordere und hintere Wand zum Vorschein kamen; von den 2 Patientinnen, bei denen Heilung durch Kolporrhaphia anterior duplex erzielt worden war, die aber ein Recidiv bekommen hatten, betraf das eine einen nochmaligen Prolaps des Uterus mit Portioelongation, das andere Mal war durch den bei der Geburt angelegten Forceps der Damm wieder eingerissen, die hintere Wand stark prolabirt, während die vordere, bei ruhigem Zustande sichtbar, erst beim Pressen etwas prolabirte. Also wir sehen, dass entschieden durch die Kolporrhaphia duplex doch weit günstigere Resultate betreffs Verhinderung eines Recidives der vorderen Wand erzielt wurden, als durch die Kolporrhaphia anterior simplex.

Was weiterhin den Operirten zum. Vortheil zu gereichen scheint, ist die Ausführung der Operation in zwei Zeiten. Abgesehen dayon, dass die Frauen längere Zeit von ihrer oft schweren Arbeit befreit sind und sich meist auch durch den längeren Spitalaufenthalt das Allgemeinbefinden bei guter Kost und Pflege hebt; was gewiss auch auf die gute Heilung einen Einfluss ausübt, so wird 
durch die einzeitige Operationsmethode plötzlich eine so grosse Veränderung der Gewebstheile zu einander geschaffen, dass deren Ernährung naturgemäss darunter leiden muss; lässt man aber dem einen Theil der Scheide Zeit zur Heilung und Rückbildung, während der andere Theil noch seine gleichen Ernährungsbedingungen besitzt, so dient dies gewiss nur zum Vortheil der Operation und ermöglicht eine raschere Heilung.

Es hat dies gewiss mit dazu beigetragen, dass das Resultat der Basler Klinik um 20 pCt. günstiger ausgefallen ist, als das der Halle'schen Klinik; neben diesem Umstand aber verdanken wir diesen Vortheil zum grossen Theil auch der Einführung der Kolporrhaphia anterior duplex; und die Erfahrungen, die wir damit gemacht haben, sind günstig genug, um darauf gestützt die Kolporrhaphia anterior duplex Fehling in schweren Fällen von Prolapsus uteri et vaginae und auch in solchen von Prolapsus vaginae allein warm empfehlen zu können, und es gebührt ihr jedenfalls in der Reihe der als vortheilhaft bewährten Prolaps-Operationen ein hervorragender Platz. 